\title{
Zwei neue Arten Inocybe aus dem Ostsee-Raum
}

\author{
JOHANN STANGL und HORST GLOWINSKI
}

\begin{abstract}
STANGL, J. \& GLOWINSKI, H. 1981: Zwei neue Arten Inocybe aus dem OstseeRaum. - Karstenia 21: 26-30.

Die neuen glattsporigen Arten Inocybe hygrophana Glowinski \& Stangl und I.pseudoreducta Stangl \& Glowinski werden beschrieben aus der Bundesrepublik Deutschland. I. hygrophana: Klein. Durchfeuchtet Hut sepiafarben mit violetter Randzone, Lamellen auffallend hell violett, Stiel jung hell blauviolett und nur oben bereift. Trocken Hut ocker-rostfarben, Lamellen bräunend, Stiel kleinknollig und bräunend. I. pseudoreducta: Mittelgross mit lief rötlichbraunem Hut, mil jung zitronfarbigen, alı lichtbraunen Lamellen und mit gerandet knolligem, völlig bereiftem, zartockerlichem bis rötlichbehauchtem Stiel.
\end{abstract}

STANGL, J. \& GLOWINSKI, H. 1981: Two new species of Inocybe from the Baltic region. - Karstenia 21: 26-30.

\begin{abstract}
The new smooth-spored species Inocybe hygrophana Glowinski \& Stangl and I. pseudoreducra Stangl \& Glowinski are described from the Feredal Republic of Germany. I. hygrophana: Small. When moist, cap sepia brown with a violet marginal zone, gills distinctly light violet, and stipe light bluish violet and pruinose above when young. When dry, cap ochraceous-ferruginous, gills brownish, and stipe bulbillous and brownish. I. pseudoreducta: Medium-sized, cap deep brown with a reddish tint, gills citrine at an early stage, light brown when mature. Stipe marginate-bulbous, totally pruinose, faintly ochraceous to reddish.
\end{abstract}

Johann Siangl, Von den Tann-Sir. 48, D-8900 Augsburg, Deutschland Horst Glowinski, Bei der Wasserkunst I, D-2400 Lubeck, Deutschland

\section{Einleitung}

Der Fundort der neuen Risspilze Inocybe hygrophana und $I$. pseudoreducta liegt in Müggenbusch bei Lübeck, Bundesrepublik Deutschland, eng beisammen. Müggenbusch liegt an der Wakenitz und war früher nach den anderen Seiten hin von einem wasserreichen Erlenbruch (hauptächlich Alnus glutinosa) begrenzt. Seit einigen Jahrzehnten ist Müggenbusch mit dem festen Lande durch eine Wegaufschüttung von Bauschutt, Kies, Humus usw. verbunden und genau am Anfang dieses Verbindungsweges liegt unmittelbar vor der Gaststätte Müggenbusch in einer kleinen Neuanpflanzung unser Erstfundort.

Inocybe hygrophana, die inzwischen auch an einer weiteren Stelle in der Nähe von Müggenbusch (St. Hubertus) aufgefunden werden konnte, wuchs auf nacktem, zuweilen mit niedrigen Moosen bewachsenem, dünnschichtigem Humusboden bei Alnus, Betula, Salix und zwar gesellig wie auch öfter in kleinen Büscheln. Diese Art ist unseres Wissens bisher in Europa noch nicht bekannt gewesen. So sucht man auch bei Heim (1931), Kühner (1955) oder Kühner und Romagnesi (1953) vergeblich nach vergleichbaren Arten. Im Bestimmungsbuch von Moser (1978) wird zwar I. vatricosa (Fr.) Karst., die auch stark hygrophan sein soll und die wir selbst nie gesehen haben, angeführt, aber sie hat doch nach der Beschreibung völlig andere Merkmale. Schliesslich haben wir den Namen hygrophana gewählt, weil unser Fund eine bei Inocybe ungewöhnliche Hygrophanität aufweist, wie sie so nur bei den Genera Psathyrella und Cortinarius (Untergattung Telamonia) vorzufinden ist. Auf Grund der violetten Farbtöne bei jungen und durchfeuchteten Exemplaren möchte sich taxonomisch eine Einordnung bei den Lilacinae Heim empfehlen. Ob das jedoch sinnvoll ist oder ob u.U. sogar wegen der Hygrophanität die Schaffung einer neuen Untersektion Hygrophanae mit den Arten I. vatricosa und I. hygrophana noch empfehlenswerter 
sein möchte, das mag auf dem Wege zu einem natürlichen System einer späteren Klärung vorbehalten bleiben.

Was aber I. pseudoreducta anbetrifft, die gesellig oder einzeln, im Gras oder auf Nadelstreu wachsend unter einer jungen Fichtenhecke gefunden wurde, so dürfte für sie eine gewisse Kalkanteiligkeit im Boden und als Wirtpflanze das Vorhandensein von Nadelbäumen (Fichten) erforderlich sein.

Übrigens hat Lange (1935-1940) seine I. reducta original beschrieben und dieser Beschreibung eine Abbildung beigegeben. Er vermerkt dort betr. der Mikro-Merkmale: 'Spores minute, ovate-ellipsoid, $6-7 \times 4 \mu \mathrm{m}$. Cystidia rather short, bottle-shaped, crested (about $60 \times 12-14 \mu \mathrm{m}$ ), both on the sides and the edge of the gills. Fibrils on cap $3-5 \mu \mathrm{m}$ broad.' und fügt dann im Kleindruck hinzu, dass seine reducta im Wuchs der I. umbrina ähnlich sei, aber auch an Kleinformen der I. asterospora erinnern möchte, wiewohl sie weniger rissig sei. Nun, alle diese von Lange vergleichsweise herangezogenen Arten incl. I. microspora und I. descissa kennen wir schon seit Jahren und sind zu dem Ergebnis gekommen, dass sich nicht nur die $I$. reducta, sondern auch unsere $I$. pseudoreducta makroskopisch wie mikroskopisch gut von ihnen unterscheiden lassen. Inzwischen haben wir auch den von Engel \& Engel (1980) beschriebenen Fund der I. reducta revidiert und die von Lange angegebenen Mikro-Werte wiedergefunden, Ein eigner Fund der I. reducta, auf den wir zufällig bei St. Hubertus (etwa $2 \mathrm{~km}$ von Müggenbusch), am 4. VIII. 1980 gestossen waren und der wohl neben dem Engel'schen Fund in Süd-OstHolstein einer der wenigen Funde im baltischen Raum überhaupt sein dürfte, hatte die gleichen vorgegebenen Mikro-Werte und konnte ebenfalls mit unserer $I$. pseudoreducta verglichen werden. Es ergab sich dabei, dass sich 1 . pseudoreducta in folgenden Punkten von $I$. reducta unterscheidet: (1) durch den völlig bereiften Stiel, (2) durch die grossen Sporen, (3) durch zumindest kompaktere Zystiden und (4) möglicherweise auch durch die exklusive Bindung an Picea, zumal sie sich am Fundort nur an Picea hielt und Laubhölzern augenscheinlich aus dem Wege ging. - Um auf eine mögliche Verwechslung mit $I$. reducta hinzuweisen, haben wir für unsere Inocybe den Namen pseudoreducta gewählt.

Inocybe hygrophana Glowinski \& Stangl sp.n.

Pileus $1.5-3 \mathrm{~cm}$ in diam., verrucose umbonatus, valde hygrophanus, in statu imbuto sepia-brunneus, in statu sicco ochraceus et saepe etiam ferrugineo permixtus, persubtiliter tomentosus, circum marginem crassius tomentosus usque subfibrillosus, nunquam squamulosus. Lamellae potius subconfertae, usque ad $4 \mathrm{~mm}$ latae, adnexae vel paulum liberae, primum insigniter caeruleoviolaceae, tum sordide ochraceae vel pallide brunnescentes. Stipes 2-3 cm altus, $0.2-0.5 \mathrm{~cm}$ latus, plerumque aequalis, caeruleo-violaceus, denique pallide brunneus, superne pruinosus, subglaber et substriatus. Caro alba, in superiore parte stipitis tamen tenuiter violaceo-afflata, odore spermatico.

Basidiosporae 8-10-10.5 $\times(5-)$ 5.5-6.5 $\mu \mathrm{m}$, ventricosae, ovales. Cheilocystidia 45-70 (-80) $\times 14-21$ $\mu \mathrm{m}$, muricata, membranis tenuibus, in $\mathrm{NH}_{4} \mathrm{OH}$ lutea. Pleurocystidia 50-80 (-95) $\times 12-18 \mu \mathrm{m}$, om:nino sicut cheilocystidia. Caulocystidia solum superne, 50-80-90 × (8-) 10-20 $\mathrm{mm}$, plurimum muricata, membranis tenuibus. Epicutis pilei e hyphis usque ad $13 \mu \mathrm{m}$ latis. Habitatio: sub alnis, betulis et salicibus in propinquitate picearum juvenilium.

Hut: $1.5-3 \mathrm{~cm}$ breit und $0.5-0.8 \mathrm{~cm}$ hoch; jung konisch gewölbt mit etwas vorgezogenem Scheitel, bald flach gewölbt bis scheibenförmig mit mehr oder weniger vorstehendem, warzigem Buckel, alt wellig verbiegend, Cortina sehr flüchtig: Rand jung etwas eingebogen, bald abgebogen, schliesslich scharf abstehend und meist etwas hochgeschlagen. Farbe: durchfeuchtet dunkel sepia-braun, Randzone mit violettlichen Tönen und lange beim Austrocknen dunkel bleibend, trocken schmutzig ockerfarben mit überlagerten rostfarbenen Tönen, aber gelegentlich auch nur lederfarben-ockerlich. Hutbedeckung im Scheitelbereich mit spärlichen, grauen Velumresten, die auf einem sehr feinem Filz liegen, zum Rande feinst filzig (Lupe) und am Rande gröber filzig bis minimal faserig werdend. Nie schuppig, auch nicht andeutungsweise. Lamellen normal stehend, eher etwas gedrängt, untermischt bis $4 \mathrm{~mm}$ breit, halbbogig und hakig angewachsen bis fast frei; jung auffallend hell blau-violettich, trocken und älter graubeige bis hellbraun; Schneide glatt und weissbewimpert. Stiel $2-3 \mathrm{~cm}$ hoch, $0.2-0.5 \mathrm{~cm}$ breit; zylindrisch, gleich dick oder bisweilen oben etwas verdickend und zum Grund hin etwas verjüngt, manchmal aber auch an der Basis angeschwollen und kleinknollig; jung hell blauviolettlich, alt bräunend; oben bis zu $0.2-0.5 \mathrm{~cm}$ fein bereift, zur Basis hin kahl erscheinend, aber unter der Lupe doch mit ganz feinen Faserflöcken zerstreut bedeckt, etwas riefig 


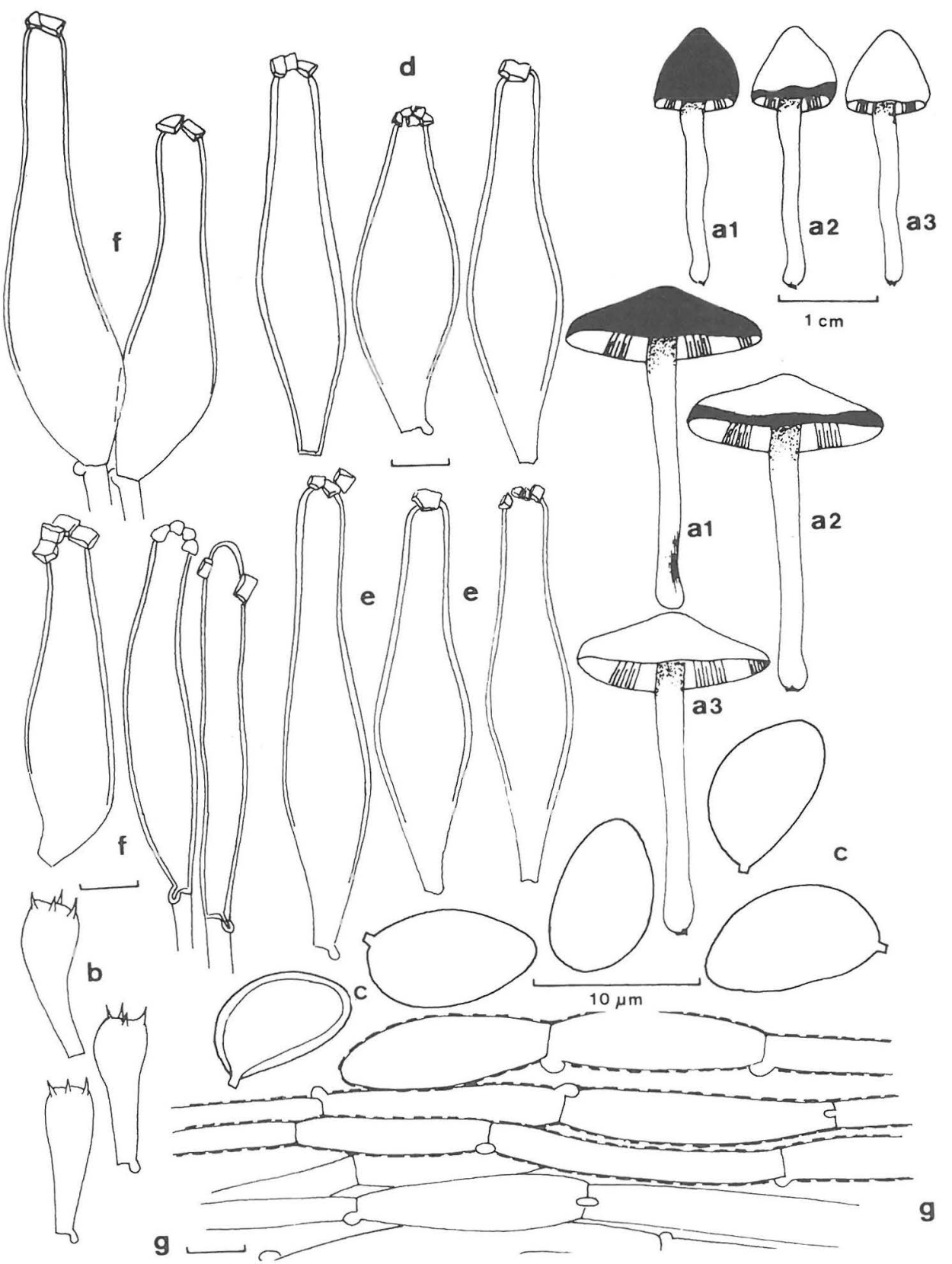

Fig. 1. Inocybe hygrophana. a) Fruchtkörper (a1 durchfeuchtet, a2 halbtrocken, a3 trocken), b) Basidien, c) Sporen, d) Cheilozystiden, e) Pleurozystiden, f) Kaulozystiden, oben, g) Hyphen der Hutbedeckung.

erscheinend Fleisch im Hut weiss, $1-1.5 \mathrm{~mm}$ dick, durchfeuchtet hyalin. Im Stiel violettblau, im oberen Stieldrittel sich lange so haltend und zur Basis hin verblassend, fein faserig. Geruch mehr oder weniger stark spermatisch. Sporenstaub tabakfarben.

Basidien 27-30 $\times 8-10 \mu \mathrm{m}$, vorwiegend mit 4 


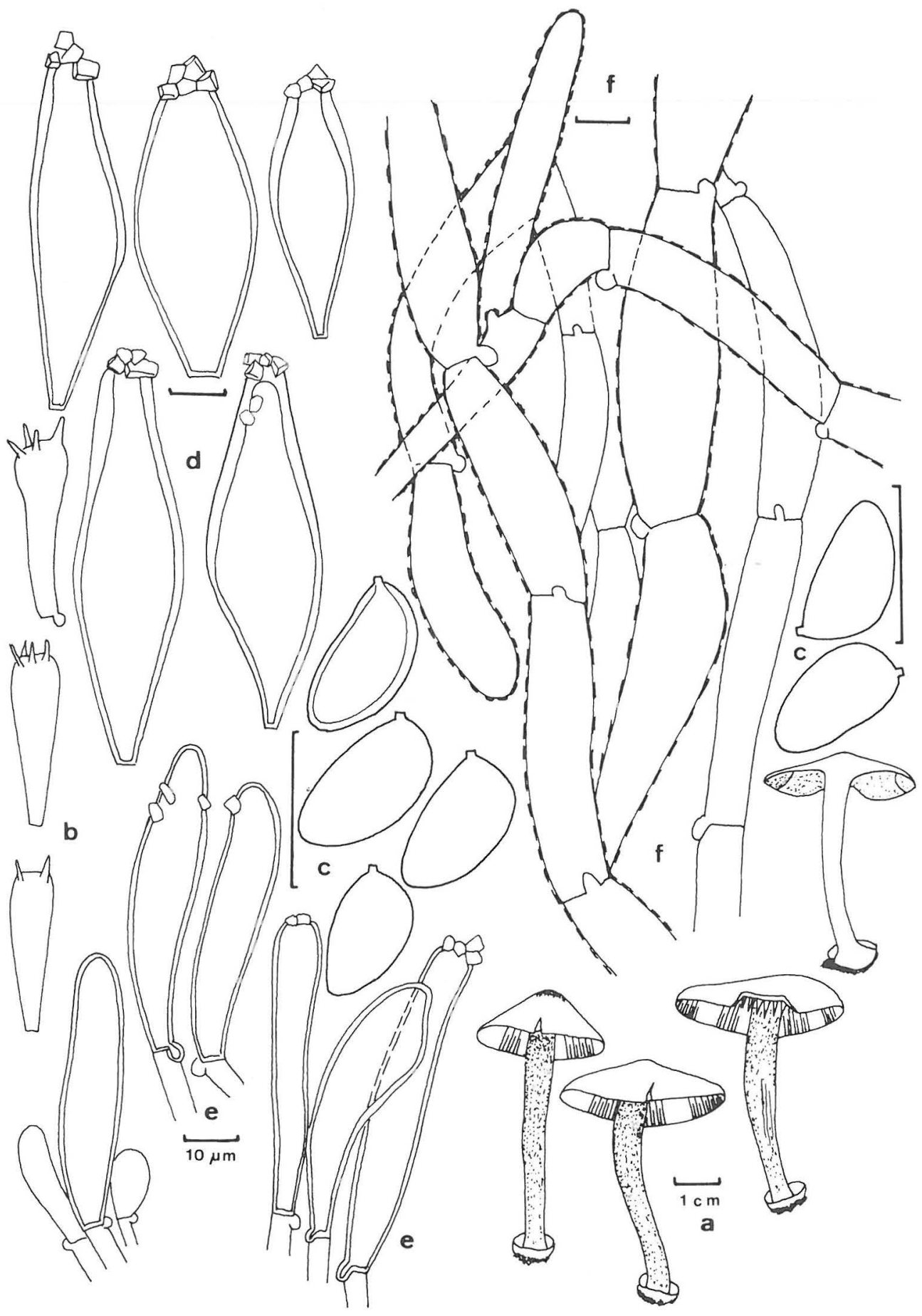

Fig. 2. Inocybe pseudoreducta. a) Fruchtkörper, b) Basidien, c) Sporen, d) Hymenialzystiden, e) Kaulozystiden, unten, f) Hyphen der Hutbedeckung. 
Sterigmen. Basidiosporen 8-10-10.5 $\times$ (5-) 5.5-6.5 $\mu \mathrm{m}$, bauchig, oval. Cheilozystiden 45-70 (-80) $\times 14-21 \mu \mathrm{m}$, mit Kristallen, dünnwandig, in $\mathrm{NH}_{4} \mathrm{OH}$ gelb. Pleurozystiden 50-80 (-95) $\times$ $12-18 \mu \mathrm{m}$, mit Kristallen, dünnwandig, in $\mathrm{NH}_{4} \mathrm{OH}$ gelb. Nich auffällig verschieden von den Cheilozystiden. Kaulozystiden nur ganz oben, 50-80-90 $\times(8-) \quad 10-20 \mu \mathrm{m}$, dünnwandig. Epikutis aus Hyphen, die bis $13 \mu \mathrm{m}$ breit werden.

Holotypus: Bundesrepublik Deutschland, Müggenbusch prope Lübeck, MTB 2130, 16. VIII. 1980 H. Glowinski (Herb. M).

\section{Inocybe pseudoreducta Stangl \& Glowinski} sp.n.

Pileus 3-5.5 cm in diam., conice convexus, dein plane convexus usque expansus, obtuse et late umbonatus, particulis terrae obtectus, vertice lanato, satis brunneo vel fusco-rubro, marginem versus sature brunneo-fibrillosus in fundo ochraceobrunneo usque argillaceo-ochraceo; cortina non visa. Lamellae plus minusve confertae, adnatae, conspecte sordide citrinae, postremo sordide brunneolae, usque $0.7 \mathrm{~cm}$ latae, acie albo-fimriatae. Stipes $3.5-6 \mathrm{~cm}$ altus, $0.4-0.6(-0.7) \mathrm{cm}$ latus, aequalis vel etiam interdum ad basim subattenautus, bulbo albo, manifeste marginato et usque $1.2 \mathrm{~cm}$ lato, tenelle incarnatus, ab apice fere usque ad basim dense, sed decrescenter pruinosus, striatus, sericeo nitidus. Caro alba in apice stipitis et in bulbo, alibi incarnate tincta, omnino subaquosa, odore acidulo.

Basidiosporae 9-11.5 × 5.5-6 $\mu \mathrm{m}$, ovales vel amygdaliformes, distincto apiculo. Cystidia hymenii 40-70 $\times 17-25 \mu \mathrm{m}$, membranis tenuibus, sed in collo usque $2.5 \mathrm{~cm}$ crassis et in $\mathrm{NH}_{4} \mathrm{OH}$ luteis. Caulocystidia basi 37-60 × 10-19 $\mathrm{mm}$, tenuiter tunicata. Epicutis pilei e hyphis oblongis usque $25 \mu \mathrm{m}$ crassis et copiose fibulatis. Habitatio: congregatim et singillatim sub dumeto picearum juvenilium in gramine et in stramento acuum.

Hut: $3-5.5 \mathrm{~cm}$ in Durchmesser, jung konisch gewölbt mit abgerundetem Scheitel, bald sich ausbreitend, flach konisch werdend, mit abgerundetem, breitem und mit Erdpartikelchen bedecktem Buckel. Rand jung schwach eingerollt, ohne sichtbare Cortina, bald winklig abgebogen, alt scharf abstehend und wenig einreissend. Hutfarbe: Jung tiefbraun mit rötlichen Beitönen und deutlich durchscheinender ockerlicher Grundfarbe, alt am Scheitel sattbraun, zum Rande hin ockerlich-braun und bisweilen auch lehmfarben aufhellend. Hutbedeckung: Scheitel wollig, zum Rande hin jung dicht und fein befasert, dann gröber und aufgelockert-faserig besonders im Randgebiet. Lamellen: Eher etwas gedrängt, untermischt, bis 0.7 $\mathrm{cm}$ breit, mehr oder weniger $1 / 4$ bogig und hakig angewachsen; jung zitronfarben mit schmutzigen Beitönen, alt schmutzig beige bis lichtbraun, etwas olivlich, mit glatter, weissbewimperter Schneide. Stiel: $3.5-6 \times 0.4-0.6(-0.7) \mathrm{cm}$, zylindrisch, gleichdick oder oben verdickt und unten etwas verdünnt, mit weisser, deutlich abgesetzter, berandeter Knolle, die bis $1.2 \mathrm{~cm}$ breit und $0.7 \mathrm{~cm}$ hoch werden kann. Die zart fleischfarbenen, zart ockerlichen Stiele sind mehr oder weniger rötlich angehaucht und bis unterhalb der Mitte dicht bereift. Dieser Reif nimmt zur Basis hin ab und kann auch übersehen werden. Leicht seidig glänzend. Fleisch im Hut weiss und dort bis $2.5 \mathrm{~mm}$ dick; im Stiel oben und auch in der Knolle weiss, sonst zart fleischfarben oder wässerig-braun getönt, faserig. Geruch deutlich säuerlich. Sporenstaub tabakfarben.

Basidien 28-33 $\times(7-) 8-10 \mu \mathrm{m}$, vorwiegend mit 4 Sterigmen. Basidiosporen 9-11.5 $\times$ 5.5-6 $\mu \mathrm{m}$, oval bis mandelförmig, mit deutlichem Apikulus. Hymenialzystiden $47-70 \times 17-25 \mu \mathrm{m}$, im Hals mit bis $\mathrm{zu} 2.5 \mu \mathrm{m}$ dicken Wänden, die nach unten hin dünn werden und sich in $\mathrm{NH}_{4} \mathrm{OH}$ gelb färben. Pleuro- und Cheilozystiden unterscheiden sich voneinander kaum. Kaulozystiden $37-60 \times 10-19$ $\mu \mathrm{m}$, im unteren Stieldrittel, dünnwandig. Hyphen der Hutbedeckung bis $23 \mu \mathrm{m}$ breit und reichlich mit Schnallen versehen.

Holotypus: Bundesrepublik Deutschland, Müggenbusch bei Lübeck, MTB 2130, 2. VII. 1980 H. Glowinski (Herb. M).

\section{Literatur}

Engel, M. \& Engel, H. 1980: Inocybe reducta Lge. im südlichen Holstein. - Westfälische Pilzbriefe 11: $184-185$.

Heim, R. 1931: Le genre Inocybe. - 431 pp., 35 pls. Paris.

Huijsman, H. 1955: Observations on Agarics. - Fungus 25: $18-43$.

Kühner, R. \& Romagnesi, H. 1953: Flore analytique des champignons supérieurs. - $557 \mathrm{pp}$. Paris.

Kühner, R. 1955: Compléments à la Flore analytique 5. Inocybe leiosporés cystidiés. - Suppl. Bull. Soc. Nat. Oyonnax 9: 3-95.

Lange, J. 1935-1940: Flora Agaricina Danica. - 515 pp. Copenhagen.

Moser, M. 1978: Die Röhrlinge und Blätterpilze: In: Kleine Kryptogamenflora II b 2, Basidiomyceten 2. - 532 pp. Stuttgart.

Angenommen zur Publikation

4. Februar 1981 